\title{
Discussion on the importance of sports psychology to the training of basic fire fighters
}

\author{
Chuansong $\mathrm{Wu}$
}

Public Security Fire Force College, Kunming 650208, China

Keywords: Grass roots firefighting; psychology; warrior training

\begin{abstract}
From the macro perspective describes the importance of sports psychology of grass-roots fire fighters, the analysis of the basic psychological state, fire fighters training exercise on the significance, train fire fighters fire fighters psychological quality in three aspects. The analysis and evaluation of primary fire fighters sport psychological status, and make some suggestions for the study of fire fighters grassroots sports psychology, so as to provide some thoughts on the theory and practice of grassroots fire fighters movement psychology research in China, in order to improve the quality of basic psychological fire fighters.
\end{abstract}

\section{Introduction}

The research object of this paper is the basic level fire fighter, mainly involving two aspects: fire fighters, training psychological state and training the psychological quality of fire fighters.

\section{Research method}

Experimental research methods, laboratory experiments, natural experiments, quasi experiments, field research methods, psychological measurement, etc.

\section{The main training psychology of grass roots fire fighters}

From the characteristics of firefighting, the factors that affect the training status of fire fighters include age, physical fitness, intelligence and experience, etc. According to the relevant experiments and information, there are several mental states

\subsection{Negative state}

The fire fighters have to avoid negative attitude towards training, training that has no meaning, not distracted, do not trust, for their fear of physical quality training, especially the fear of failure game training courses, not timely adjust their psychological state, even when not to participate in training. In a sense, it belongs to no fighting will or negative psychological state.

\subsection{Too excited}

Fire fighters excessive excitement, irritable mood, attention is not concentrated, everything around the special care, sensory and perceptual features weakened, but also lead to frequent urination and other phenomena.

\subsection{Blind confidence}

Fire fighters overestimate their strength, their ability to focus, think, feel, and decide, and their responses are relatively slow. 


\subsection{Positive}

Fire fighters look forward to training, and feel the training is happy, clear understanding of training, the training is full of desire, have a clear understanding of their own strength, and can smooth, stable completion of training.

\section{Significance of sports to fire fighters}

\subsection{Influence on metabolism}

Physical training can increase the uptake and utilization of sugar by tissue cells in vivo, and enhance the storage of liver glycogen and muscle glycogen. Physical fitness also improves the body's ability to regulate glucose metabolism. The fat content of more energy in the body, it releases energy in vivo oxidation, is about two times of the same amount of sugar or protein, long-term physical training can improve the body's ability to use fat, provide more energy sources for human activities.

\subsection{The impact on the movement system}

Insist on physical training, will have a good effect on the muscles, bones, ligaments and joints, often participate in physical exercise can maintain normal muscle tension, and through continuous muscles to bone tissue to stimulate, promote bone calcium storage, preventing osteoporosis, at the same time to make the joint to maintain good flexibility, ligament keep the good elasticity, physical training can improve the accuracy and consistency of the motor system, and keep flexible, can make people relaxed, normal to do complex actions.

\subsection{Effects on the cardiovascular system}

Exercise is the only way which must be passed to heart health, with regular exercise, can slow down the static idle time and heart rate during exercise, which can greatly reduce the heart work time, increase cardiac function, maintain the coronary artery blood flow, which can supply better myocardial needed nutrition, can make the risk of heart disease the rate of reduction.

\section{4 effects on the respiratory system}

Regular physical exercise, especially stretching and stretching, can increase the strength of the respiratory muscles.

Physical training can increase the breathing depth by strengthening the breathing force, so as to effectively increase the ventilation efficiency of the lungs.

\subsection{Effects on the digestive system}

Physical training will accelerate the process of the energy consumption of the body, the ultimate source of energy material is obtained through the ingestion of food, therefore, after exercise will promote the change of function of the digestive system increased appetite, strengthen the digestive function.

\subsection{Effects on the central nervous system}

Physical fitness training can improve the nervous system regulation function, improve the nervous system on human activities of the complex changes in the ability to judge, and timely coordination, accurate and rapid response. 


\section{Cultivate the psychological quality of soldiers}

\subsection{Different from each other, targeted training}

Psychology holds that the external manifestations of each person's psychological characteristics are different. Therefore, according to different personality, targeted training of random awareness and resilience. The quick, sensitive, but brash, careless warrior, attention should be paid to play in battle they thought agile, quick advantage, focus on training their ability of thinking, careful observation, to prevent simple impatience, recklessly to other emotions; emotion has great adaptability, rapid response and flexible however, lack of perseverance, work more hasty fighters, they should play the adaptability, quick reaction, focus on the cultivation of their strong willpower and in a variety of difficult conditions out of danger will prevent hasty; for calm, slow, lack of passion in the warriors, encourage them calmly at the same time, through intelligence training, focus on training their ability to respond and deal with the sensitivity of the change of things; for with a strong willpower and good inhibition Ability, but more rigid and unresponsive warriors, should play their self-control ability and indomitable spirit, focus on the cultivation of its flexible and fast intense fighting ability, more encouragement, less blame, so it has advantages, shortcomings.

\subsection{Strengthen physiological training, improve physiological endurance}

The difficulty, complexity and danger of fire disposal process have high demands on the physiological and psychological qualities of fire fighters. Fire fighters can adapt to the mental condition of complex working conditions and their physical quality also have a certain relationship, the physical quality is better, often fire fighters psychological ability is stronger in fire fighting, nervous tension is small. Therefore, in order to enable fire fighters to withstand excessive intensity of physiological and psychological pressure in fire fighting, physical training should be strengthened. To improve the flexibility and psychological stability of the fire fighter's limbs, physical training is necessary.

\subsection{Outstanding technical and tactical training, to improve the adaptability of soldiers}

According to the psychological characteristics of response and fire fighting fire fighters, combined with the characteristics of equipment, to the scene as the premise, bring the technology and tactical training, to practice skills, training, training with the ability to adapt as the focus, to carry out targeted training. Can also be appropriate to combine the local fire rescue mission in reality, and gradually carry out test tactical drills, through actual combat training, testing soldiers in actual combat conditions of fire fighting ability.

\subsection{Strengthen actual combat simulation training}

Actual combat simulation training is one of the most effective ways to improve the psychological quality of fire fighters. Mainly from the following aspects:

By assuming a dangerous environment, increase the courage of fire fighters.

Let fire fighters train themselves at high temperatures to hone their will.

Carry out necessary simulated fire danger training.

\subsection{Strengthen the psychological training of team spirit of fire fighters}

In a variety of fire fighting and emergency rescue, often encountered by a small number of panic caused by a chain reaction, this phenomenon is known as "psychological infection."". Carrying forward team spirit is the key to accomplish all tasks. It is also the purpose of strengthening the training of team and team in training. The psychological training of fire fighting collective is through the practice of strengthening the ideological and political education, the theoretical knowledge of the business training and fire extinguishing tactics combining the training of fire fighters collective 
concept, discipline and the spirit of unity and cooperation, strengthen the psychological self-control ability.

In short, the good psychological quality of fire fighters requires long-term and repeated training to gradually form and consolidate. Psychological quality training should be gradual, training content from simple to difficult, not anxious, according to the physiological and psychological characteristics of the fire fighters, with proper training contents and methods for fire fighting and rescue requirements, in order to obtain the best training effect.

\section{References}

[1]Arnold, LeUnes, Jack, R. Nation. Introduction to exercise psychology. Third edition. Yao, Chinese translation, Shaanxi Normal University press, 2005.

[2] Mao Jie. Sports psychology, East China Normal University press, 2003.

[3] Mao Jie. Research progress in sports psychology, higher education press, 2000. 